\title{
Research on the Tensile Properties of Packaging Film Based on Environmental Protection PVA-KGM
}

\author{
Ligui $\mathrm{XIONG}^{1}$ and Liqiong ZHANG \\ Guangdong Polytechnic Vocational College, Zhongshan, Guangdong, China
}

\begin{abstract}
Konjac glucomannan (KGM) and poly (vinyl alcohol) (PVA) were mixed to form gel-like polyelectrolyte solution with glycerol and sorbitol as compound plasticizer, which was used to prepare packaging films via casting and drying. The results show that the tensile strength and elongation at break of the packaging films drop sharply when the blending temperature and blending time exceed $80^{\circ} \mathrm{C}$ and $3.5 \mathrm{~h}$. When the mass ratio of sorbitol and glycerol in the compound plasticizer ranges from $1: 1$ to $1: 3$, it is beneficial to improve the tensile strength of the packaging films. The microscopic reasons for the change of the tensile properties of the packaging films are mainly caused by diffusion-stop-continuing diffusion - precipitation of low-molecular electrolyte, which makes the system shift from equilibrium-homogeneous stateunbalanced state - heterogeneous state.
\end{abstract}

Keywords. Konjac glucomannan, poly (vinyl alcohol), packaging films, environmental protection

\section{Introduction}

KGM is mainly extracted from the natural tuber herb konjac. It is a natural polysaccharide water-soluble dietary fiber with film-forming and biodegradability, and can be used to prepare environmentally friendly polymer materials [1-5]. PVA is nontoxic, odorless, and harmless. It is the only vinyl polymer that can be used by bacteria as a carbon source and energy source. Under the action of bacteria and enzymes, PVA can degrade $75 \%$ in 46 days. It is a kind of biodegradable polymer material. It can be mass-produced by non-petroleum route and is low in price. The film made is flexible and smooth, oil-resistant, solvent-resistant, and abrasion-resistant. Good gas permeability and damage [6-10]. The molecular structure of PVA contains a large number of hydroxyl groups, and strong hydrogen bonds are easily formed within or between molecules, resulting in a higher melting point, narrow melting temperature range, and difficult processing. The KGM molecule is a macromolecular aggregation structure, which contains a large number of hydroxyl groups, and there are hydrogen bonds between molecular chains, which is difficult to directly plasticize. In the previous research on the blending of PVA and KGM, the PVA/KGM blending system can be fully plasticized by adding a plasticizer compounded with glycerol and sorbitol (combined plasticizer for short), and the melting temperature is reduced. A packaging

\footnotetext{
${ }^{1}$ Corresponding Author, Ligui XIONG, Guangdong Polytechnic Vocational College, Zhongshan, Guangdong, China; Email: 1049661754@qq.com.
} 
film with excellent performance was prepared [11-12]. On this basis, this work further explores the factors affecting the tensile properties of packaging films.

\section{Experimental Part}

\subsection{Main Raw Materials}

Konjac powder, food grade, w(KGM) $>90 \%$, Hubei Huipu Biochemical Technology Co., Ltd.; PVA, industrial grade, Japan Kuraray Company; Sorbitol, glycerin: both analytically pure, Sinopharm Chemical Reagent Co., Ltd.

\subsection{Basic Formula and Sample Preparation}

Weigh a quantitative amount of konjac powder, add ethanol, filter, dry, and purify for use. In the previous study [11-12], select the appropriate ratio, mix the purified konjac powder and PVA according to the mass ratio of 1:10, and then dissolve it in $100 \mathrm{~mL}$ of distilled water, and add a $10 \%$ mass fraction compound the plasticizer is stirred at a constant temperature to prepare a film liquid. The film liquid is defoamed by ultrasonic and then cast on flat glass, and dried naturally to form a film. A film with a thickness of 0.3 to $0.4 \mathrm{~mm}$ is taken as the required sample.

\subsection{Performance Test Method}

The tensile properties are tested according to GB/T 1040.3-2006.

\section{Results and Discussion}

\subsection{Effect of Blending Temperature on the Tensile Properties of Packaging Film}

The purified konjac powder was blended with PVA for $3.0 \mathrm{~h}$, the mass ratio of glycerin to sorbitol in the compound plasticizer was $2: 1$, and the blending temperature was 50 , $60,70,80$, and $90^{\circ} \mathrm{C}$, respectively.

It can be seen from figure 1 that when the temperature is $80^{\circ} \mathrm{C}$, the tensile strength and tensile strain at break of the packaging film both reach their peaks, and as the temperature continues to rise, both decreases. The viscosity of a single-component PVA aqueous solution decreases significantly with the increase of temperature, but after adding KGM and PVA to blend into a gel-like polyelectrolyte solution, it dissociates into polymer ions and hydroxyl groups under the action of water. When the temperature increases, various ions as the activity frequency increases, the electrostatic attraction and repulsion effects in the system also increase. When the blending temperature reaches $80{ }^{\circ} \mathrm{C}$, various forces form a stable equilibrium state, the polymer molecular chains are entangled, and the mutual displacement between molecules is difficult., The flow resistance increases, the viscosity of the system is the largest, the tensile strength and tensile strain at break of the packaging film are the largest, the brittleness of the packaging film is reduced, and the toughness is increased. When the blending temperature continues to increase, the frequency of various ionic activities 
increases again, destroying the equilibrium state of the blending system, but the polymer chain segment cannot overcome the energy barrier in time to move during the transition. Therefore, the stretching of the packaging film the strength and tensile strain at break began to drop sharply.

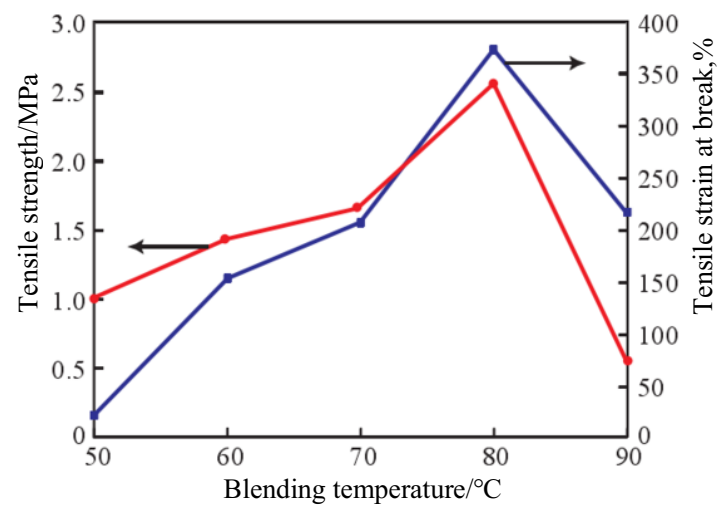

Figure 1. Blending temperature as a function of tensile properties of packaging films.

\subsection{The Effect of Blending Time on the Tensile Properties of Packaging Film}

The purified konjac powder and PVA are blended at a water bath temperature of $80^{\circ} \mathrm{C}$. The mass ratio of glycerin to sorbitol in the compound plasticizer is $2: 1$, and the blending time is $2.5,3.0,3.5,4.0$, respectively. $4.5 \mathrm{~h}$. The blending time determines the time required for the blending of PVA and KGM to form a homogeneous system of gel-like polyelectrolyte solution.

It can be seen from figure 2 that the tensile strength and tensile strain at break of the packaging film are both maximum when blended for $3.5 \mathrm{~h}$. This is because PVA and KGM are in an aqueous solution. Because water molecules and other small molecules (such as hydroxyl and plasticizer ions, etc.), electrolytes gradually bond with polymer ions during mutual penetration and movement, PVA and KGM are networklike cross-linked. The volume of the macromolecules gradually expands, and the swelling equilibrium is reached after 3.5 hours, the diffusion of small molecules stops, and the concentration of the small molecule electrolyte solution reaches the critical value, forming a homogeneous system. The blending time continued to increase. The small molecule electrolyte changed the molecular shape and solubility properties of the gelled polyelectrolyte formed by blending PVA and KGM, becoming a poor solvent, causing a small amount of polyelectrolyte to begin to precipitate out of the solution, and the microscopic nature is mainly low molecular the diffusion of the electrolytestop-continue to diffuse-precipitate, so that the system changes between equilibrium state-homogeneous state-unbalanced state-heterogeneous state. The macroscopic manifestation is that the tensile strength and tensile strain at break of the packaging film decrease. 




Figure 2. Blending time as a function of tensile properties of packaging film.

\subsection{The Effect of Compound Plasticizer on the Tensile Properties of Packaging Film}

The purified konjac powder was blended with PVA for 3.5 hours, the temperature of the water bath was $80^{\circ} \mathrm{C}$, and the mass ratios of sorbitol and glycerin in the compound plasticizer were $1: 1,1: 2,1: 3,1: 4,1: 5$. Sorbitol and glycerin mainly play the role of external plasticization, that is, the viscosity of the combination of the two is several or even ten orders of magnitude lower than that of the PVA/KGM blend system. When small molecules are mixed into high polymers, the viscosity will be reduced to $1 / 1000$ of the original when the component mass fraction changes by $20 \%$, which is conducive to processing. With the increase in the amount of plasticizer added, the miscibility of the blended system is enhanced, and the uniform dispersion of the phases in the system is promoted.

The mechanical properties depend on the miscibility and dispersion state of each component. It can be seen from figure 3 that as the mass ratio of sorbitol to glycerin decreases, the tensile strength of the packaging film first increases and then decreases, and the tensile strain at break first decreases and then slightly increases; the mass ratio of sorbitol to glycerin is 1:3 When the tensile strength reaches the maximum, the tensile strain at break is the smallest. This is because the added amount of plasticizer within a certain range is beneficial to reduce the viscosity of the system, increase the tensile strength and the toughness of the film, and the tensile strain at break is higher, but the mass ratio of sorbitol to glycerin continues to decrease (less than 1:3 After ), the amount of glycerin increased, which exceeded the compatibility limit of the plasticizer molecule and the macromolecule, which easily caused the migration and precipitation of the plasticizer. At this time, the surface of the packaging film also appeared "oil seepage". 


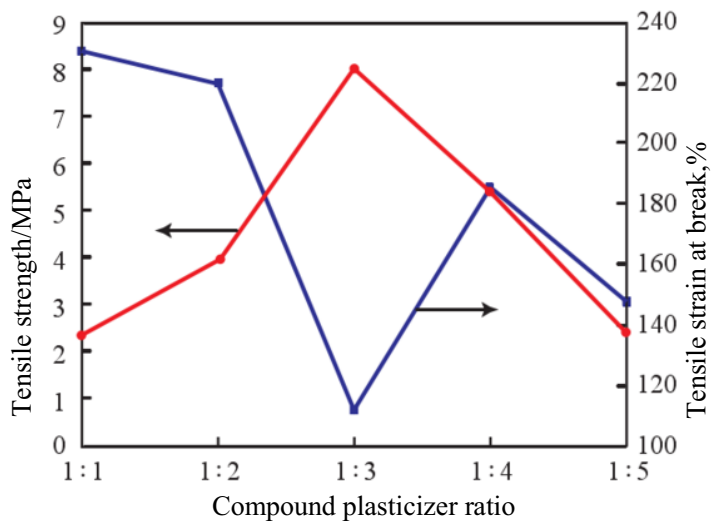

Figure 3. Proportioning of compounding plasticizer as a function of tensile properties of packaging films.

\section{Conclusion}

(1) PVA and KGM are blended to form a gel-like polyelectrolyte aqueous solution. When the blending temperature exceeds $80^{\circ} \mathrm{C}$ and the blending time exceeds $3.5 \mathrm{~h}$, the homogeneous system that has been formed can be destroyed, and the tensile strength and tensile strain at break of the packaging film after drying will drop sharply.

(2) When the mass ratio of sorbitol to glycerin in the compound plasticizer is $(1: 1)$ $\sim(1: 3)$, it is beneficial to increase the tensile strength of the packaging filmWhen the temperature is lower than 1:3, the amount of glycerin increases, which exceeds the compatibility limit of plasticizer molecules and macromolecules, and glycerin precipitation appears, the tensile strength of the packaging film decreases, and the tensile strain at break changes greatly.

(3) The reason for the destruction of the homogeneous system of PVA and KGM gel-like olyelectrolyte aqueous solution can be from the water-soluble polymer-like polyelectrolyte analysis of the characteristics of the liquid, the microscopic nature is mainly the diffusion of small molecule electrolytes - stop-continue to diffuseprecipitate, so that the system changes between equilibrium state-homogeneous state-unbalanced state-heterogeneous state. The macroscopic phenomenon is the blended membrane. The tensile properties fluctuate.

\section{References}

[1] Pang D M, Huang S L, Wu Y M, et al. 2014 Preparation and properties of hydroxypropyl cellulose/konjac glucomannan blend film New Chemical Materials 65-67.

[2] Zhang N, Sui S, Wang Y, et al. 2014 Preparation and performance of konjac glucomannanmethylcellulose edible film Food Industry Science and Technology 35 (16) 302-307.

[3] Shi Y, Liu J and Hong J 2014 Research progress on chemical modification of konjac glucomannan New Chemical Materials 42 (2) 21-23.

[4] Wang Y, Zheng X, Xie J, et al. 2006 Preparation and properties of fully degradable konjac plastic film Plastic Industry 36 (2) 60-62.

[5] Xu X, Chen H, Zheng Y, et al. 2014 Research progress of konjac glucomannan/starch composite modification Food Industry Science and Technology 33 (3) 371-375.

[6] Gao X, Liu J, Tang K, et al. 2019 Compatibility of gelatin/PVA blend packaging film Polymer Materials Science and Engineering 29 (7) 111-113. 
[7] Wang L, Chen J, Ren F, et al. 2016 Study on the melt processability of plasticizer-modified PVA Plastics Technology 40 (11) 53-56.

[8] Hu A, Gao G, Zhu W, et al. 2019 Preparation of biodegradable packaging materials by borax-urea packaging modified PVA Plastics 42 (3) 103-105.

[9] Wang Y, Chen W and Liu X 2018 Study on the film-forming characteristics of chitosan-PVA packaging film Anhui Agricultural Sciences 42 (8) 2223-2224.

[10] Zhu E, Xin M, Li M, et al. 2020 Hydrogen bonding and compatibility of chitosan/PVA blend film Chemical Industry Progress 31 (5) 1082-1087.

[11] Zhang L, Chen S, Xu J, et al. 2016 Research on light transmittance and haze of PVA-konjac glucomannan packaging film Packaging Engineering 37 (15) 84-88.

[12] Zhang L, Zhao S, Chen S, et al. 2017 The effect of glycerol/sorbitol modification on the properties of PVA-konjac glucomannan packaging film New Chemical Materials 45 (5) 218-219. 